Research Paper

\title{
KCa3.1 as an Effective Target for Inhibition of Growth and Progression of Intrahepatic Cholangiocarcinoma
}

\author{
Penghong Song1, 2*, Yehui Du1 ${ }^{1} 2^{*}$, Wenfeng Song1, 2, Hao Chen ${ }^{1,2}$, Zefeng Xuan ${ }^{1,2}$, Long Zhao ${ }^{1,2}$, Jun Chen ${ }^{1,2}$, \\ Jian Chen ${ }^{1,2}$, Danjing Guo ${ }^{1,2}$, Cheng Jin¹, 2, Yongchao Zhao ${ }^{1,2,3}$, Biguang Tuo ${ }^{4}$, Shusen Zheng1, 2® \\ 1. Key Laboratory of Combined Multi-organ Transplantation, Ministry of Public Health, First Affiliated Hospital, School of Medicine, Zhejiang University, \\ Hangzhou 310003, China; \\ 2. Collaborative Innovation Center for Diagnosis Treatment of Infectious Diseases, Hangzhou 310003, China; \\ 3. Institute of Translational Medicine, Zhejiang University School of Medicine, Hangzhou 310029, China; \\ 4. Department of Gastroenterology, Affiliated Hospital of Zunyi Medical College, Zunyi 563003, China. \\ * These authors contributed equally to this work \\ $\square$ Corresponding author: Shusen Zheng, Key Laboratory of Combined Multi-organ Transplantation, Ministry of Public Health and Collaborative Innovation \\ Center for Diagnosis treatment of infectious diseases, First Affiliated Hospital, School of Medicine, Zhejiang University, Hangzhou 310003, China. Tel: \\ 86-571-87236466; Fax: 86-571-87236466; E-mail: shusenzheng@zju.edu.cn \\ (c) Ivyspring International Publisher. This is an open access article distributed under the terms of the Creative Commons Attribution (CC BY-NC) license
} (https://creativecommons.org/licenses/by-nc/4.0/). See http://ivyspring.com/terms for full terms and conditions.

Received: 2016.12.11; Accepted: 2017.03.31; Published: 2017.06.03

\begin{abstract}
Background: Intrahepatic cholangiocarcinoma (ICC) is a high malignant tumor arising from the bile ducts in the liver with a poor prognosis. As current molecular targeted therapies and systemic chemotherapies had limited success in ICC, novel therapeutic targets are needed. In this study, we attempted to investigate the expression and the role of the intermediate conductance calcium-activated potassium channel (KCa3.1) in ICC.

Methods: The expression levels of $\mathrm{KCa} 3.1$ channel were measured in 81 resected ICC tumor specimens and the clinicopathological significance of these levels were determined. $\mathrm{KCa} 3.1$ channel inhibitor and siRNA were used to study the role of $\mathrm{KCa} 3.1$ in proliferation, migration, and invasion of ICC cell lines. The effect of KCa3.1 channel blockade on tumor growth in vivo was also studied using xenograft model in nude mice.

Results: The protein expression of $\mathrm{KCa} 3.1$ channel was upregulated in ICC tissues and was correlated with age, lymph node metastasis and TNM stage. And high KCa3.1 expression indicated a worse prognosis in ICC patients. Blocking KCa3.1 channel with a specific inhibitor TRAM-34 reduced the proliferation and invasion of ICC cells. Knockdown of KCa3.1 could achieve the same effects through decreasing NF-KB activation. Further in vivo studies demonstrated that $\mathrm{KCa} 3.1$ channel blockade suppressed ICC tumor growth.

Conclusions: Our observations suggested $\mathrm{KCa} 3.1$ might be a promising novel therapeutic target in intrahepatic cholangiocarcinoma.
\end{abstract}

Key words: KCa3.1, Intrahepatic cholangiocarcinoma, Proliferation, Invasion, TRAM-34.

\section{Introduction}

Intrahepatic cholangiocarcinoma (ICC) is a high-graded biliary malignancy arising from the small bile ducts in the liver [1]. The incidence and mortality of ICC has been rising steadily over long periods worldwide and the development of ICC is related to a wide range of conditions including cirrhosis, hepatolithiasis, liver fluke infections and hepatitis virus infection $[2,3]$. Surgical resection at early stage remains the only effective therapy but ICCs are often diagnosed at advanced stages where surgery may not be possible. Even worse, molecular targeted therapies and systemic chemotherapies had limited success in ICC compared to liver/lung/breast cancers, which is mainly resulted from limited understanding of 
molecular and pathobiological information of ICC [1]. In order to improve the efficacy of ICC therapy, novel molecular targets and the relevant therapeutic strategies of intrahepatic cholangiocarcinoma are needed.

One such potential target is KCa3.1 (The intermediate conductance calcium-activated potassium channel), member of the voltage-gated $\mathrm{K}$ (Kv) channel superfamily. KCa3.1 plays an important role not only in maintaining intracellular calcium homeostasis, but also in regulating cell volume [4]. Several studies have reported that various human cancers including glioblastoma, breast adenocarcinoma, colorectal cancer and renal carcinoma are correlated with $\mathrm{KCa} 3.1$ expression in carcinogenesis [5-8]. Besides, KCa3.1 has attracted attention as a prognostic biomarker in patients diagnosed with lung cancer or renal carcinoma [5, 9]. Owing to the important roles of $\mathrm{KCa} 3.1$ involved in cellular functions, the blocker of KCa3.1 channel has been implicated in a variety of diseases for therapeutic potential, including autoimmune diseases and tumors [10-13]. The derivative of clotrimazole TRAM-34 is a well-known blocker to study the roles of KCa3.1 channels, being much more selective and less hepatotoxic than clotrimazole [14].

In this study, we first examined the expression of KCa3.1 in ICC tumors and matched normal tissue, then studied the role of $\mathrm{KCa} 3.1$ in carcinogenesis of ICC in vitro and in vivo, and also explored the potential mechanisms related to the functional roles of KCa3.1 in intrahepatic cholangiocarcinoma.

\section{Materials and Methods}

\section{Cell lines and cell culture}

Two human ICC cell lines HUCCT1, Huh28 were utilized in this study (HUCCT1, Huh28 were obtained from the Japanese Cancer Research Resources Bank). Huh28 was cultured in RPMI-1640 containing $10 \%$ fetal bovine serum. HUCCT1 was cultured in Dulbecco's modified Eagle medium containing $10 \%$ fetal bovine serum. All cells were incubated in a humidified atmosphere of $5 \% \mathrm{CO}_{2}$ incubator at $37^{\circ} \mathrm{C}$.

\section{KCa3.1 small interfering RNA (siRNA) transfection}

Transfection of KCa3.1 siRNA was performed using Lipofectamine 2000 reagent (Invitrogen, USA) in accordance with the manufacturer's protocol. The KCa3.1 siRNA and negative control siRNA were synthesized by Origene (Origene, USA). The sequences of KCa3.1 siRNA were defined as follows: 5'-AGCCUGGAUGUUCUACAAACAUACT-3'. After transfection for 72 hours, the protein expression of KCa3.1 in Huh28 and HUCCT1 cells was detected by western blot analysis.

\section{Cell viability assay}

Cell counting kit-8 (Dojindo, Japan) was used to detect the proliferation of Huh28, HUCCT1 cells. These two cell lines were seeded in 96-well plates and placed in $37^{\circ} \mathrm{C}$ incubator to allow adherence. Then the cells were exposed to different concentrations of TRAM-34 (a specific inhibitor of KCa3.1) (Sigma, USA) for 24, 48, and 72 hours, respectively. In addition, Huh28 and HUCCT1 cells transfected with KCa3.1 siRNA or negative control siRNA were incubated for 24, 48, 72, and 96 hours after seeding in 96-well plates. On detecting time points, $10 \mu \mathrm{l}$ cell counting kit- 8 reagent was then added to each well and incubated at $37^{\circ} \mathrm{C}$ for $1 \mathrm{~h}$. Then the absorbance at $450 \mathrm{~nm}$ was measured.

\section{Analysis of cell invasion and migration}

Cell invasion and migration assays were performed using transwell chambers with pore size of $8.0 \mu \mathrm{m}$ (Millipore, USA). Filters for invasion assays were coated with Matrigel (BD Biosciences, USA) in the upper compartment before cell seeding. A total of $4 \times 10^{4}$ cells were suspended in $200 \mu \mathrm{l}$ of serum-free medium and seeded in the upper chamber, while 800 $\mu 1$ of cell culture medium supplemented with $10 \%$ FBS was added to the lower compartment. During invasion and migration assays, the cells in the upper chamber were incubated with TRAM-34 at $20 \mu \mathrm{M}$ or equal solvent (DMSO) for $48 \mathrm{~h}$ or $72 \mathrm{~h}$. Then invasive and migratory cells on the bottom surface were stained with $0.5 \%$ crystal violet. The invading or migrating cells were counted, and photographed using digital microscopy.

\section{Measurement of intracellular $\mathbf{C a}^{2+}$ concentration}

Changes in intracellular $\mathrm{Ca}^{2+}$ concentration $\left[\mathrm{Ca}^{2+}\right]_{i}$ in ICC cells were estimated using the fluorescent $\mathrm{Ca}^{2+}$ indicator Fura Red (Invitrogen, USA). Cells were seeded in $20 \mathrm{~mm}$ confocal dish and loaded with Fura Red at a final concentration of $4 \mu \mathrm{M}$ for 45 min at $37^{\circ} \mathrm{C}$ incubator in D-Hanks solution (all mmol/l: $\mathrm{NaCl}$ 135, $\mathrm{KCl} 5.9, \mathrm{CaCl}_{2}$ 1.5, $\mathrm{MgCl}_{2}$ 1.2, Hepes 11.6 and glucose 11.5 at $\mathrm{pH}$ 7.3). After incubation, the cells were washed three times with D-Hanks solution. Then cells were treated with $20 \mu \mathrm{M}$ TRAM-34 or equivalent DMSO for 2 hours. On detection, cells were exposed to calcium-free solution for 100 seconds to detect the baseline values of $\left[\mathrm{Ca}^{2+}\right]_{\text {, }}$, then the cells were superfused with D-Hanks solution containing $5 \mathrm{mM} \mathrm{Ca}^{2+}$. Fluorescence was excited at $405 \mathrm{~nm}$, and the emitted light was collected through a 
$600 \mathrm{~nm}$ narrow-band filter on Spinning Disk Confocal Microscope (DU-897D-CS0, ZEISS, Germany). The relative fluorescence intensity was used to calculate $\left[\mathrm{Ca}^{2+}\right]_{i}$. Stored images were analyzed with Image Pro Plus 6.0 software.

\section{Western blot analysis}

After different treatment above, ICC cells were homogenized in RIPA buffer (Thermo Scientific, USA) at $4^{\circ} \mathrm{C}$. After centrifugation at $14000 \mathrm{rcf}$ for 15 minutes at $4{ }^{\circ} \mathrm{C}$, the protein concentrations of supernatants were measured by the BCA protein assay (Pierce, USA). The nuclear proteins of the cells were extracted using Nuclear and Cytoplasmic Extraction Reagents (Thermo Scientific, USA) according to manufacturer's instructions. Equal amounts of proteins were separated by electrophoresis in 12\% PAGE gels (Invitrogen, USA) and then transferred onto a polyvinylidene fluoride (PVDF) membrane. The blots were blocked with 5\% non-fat milk in Tris-buffered saline containing Tween 20 (TBS-T) and further incubated with primary antibodies and secondary antibodies. The primary antibodies used in this experiment were $\beta$-actin (1:4000, ab8227, abcam), KCa3.1 (1:2000, 251868, Abbiotec), matrix metalloproteinase 2, MMP-2 (1:2000, ab92536, abcam), MMP-9 (1:1000, ab76003, abcam), Lamin A/C (1:4000, ab58529, abcam), and Phospho-NF-kB p65 (Ser536) (1:2000, 3033, CST). Immunodetection was carried out by EZ-ECL chemiluminescence detection kit (Biological Industries, Israel). Protein bands were analyzed with image analysis software, and the results were expressed relative to $\beta$-actin or Lamin A/C expression.

\section{Immunohistochemistry}

Immunohistochemical staining was performed using paired paraffin-embedded tissues from 81 ICC patients. Briefly, the tissue block was cut into $4 \mu \mathrm{m}$ sections, deparaffinized, and rehydrated. Antigen retrieval was done in $10 \mathrm{mmol} / \mathrm{L}$ citric acid buffer (pH 6.0) in a $750 \mathrm{~W}$ microwave for 15 minutes. After incubation with anti-KCa3.1 antibody (Pab27758, Abnova) at a dilution of 1:400 overnight at $4^{\circ} \mathrm{C}$, the section was washed in PBS and then incubated with secondary antibody for 30 minutes. The section was rinsed in PBS, incubated with DAB for 2 minutes, then rinsed gently in distilled water, and counterstained with hematoxylin. Negative controls were also prepared in all assays by replacing primary antibody with nonimmune rabbit antiserum. The extent of KCa3.1 staining was scored by assigning the staining area and intensity of positive tumor cells $(0$, negative; 1, weak; 2, medium; 3, high). Besides, the immunostaining of Ki-67 in mice xenograft tumors was performed using an anti-Ki67 antibody (ab92742, abcam) at a dilution of 1:500 according to the procedures above. To quantify Ki-67 protein expression, both the area and intensity of immunoreactivity were scored in five randomly selected fields.

\section{In vivo animal study}

The study was approved by the Animal Care Committee of Zhejiang University and designed in accordance with the Interdisciplinary Principles and Guidelines for the Use of Animals in Research, Testing, and Education by the New York Academy of Sciences, Ad Hoc Animal Research Committee. Huh28 cells were injected subcutaneously into the armpit of four-week-old male nude mice in a total volume of $100 \mu \mathrm{l}\left(2 \times 10^{6}\right.$ cells in PBS). Approximately 7 days after cell inoculation, mice were randomly divided into two groups: senicapoc (MCE, USA) treatment group and control group $(\mathrm{n}=10) .120 \mathrm{mg} / \mathrm{kg}$ senicapoc or solvent (DMSO) in a total volume of $50 \mu \mathrm{l}$ was injected intraperitoneally every second day. Tumor volumes for each mouse were monitored with a caliper every 2 days by measuring in two directions (length and width). The volume was calculated as length $\times$ (width) $2 / 2$. After experiment, mice were killed by cervical dislocation after narcosis with $100 \% \mathrm{CO}_{2}$. Then tumors were removed and weighed. Histological analysis was done by H\&E and the expression of Ki67 was detected using immunohistochemistry staining in these tumors.

\section{Statistical analysis}

Data are displayed as means \pm standard deviation (SD). ANOVA and Student's t-test were used to determine the statistical significance of differences between experimental groups in vitro and in vivo. The relationship between KCa3.1 expression and clinicopathological variables was assessed by the $\chi^{2}$ test. Additionally, all statistical analyses were performed using the SPSS 19.0 program for Windows (SPSS Inc., Chicago, IL, USA). A value of $P<0.05$ was taken to indicate statistical significance. Graphs were created using GraphPad Prism 5.

\section{Results}

\section{Immunohistochemical analysis of $\mathrm{KCa} 3.1$ expression and its relationship with clinicopathological parameters}

ICC is a primary malignancy derived from biliary epithelial cells within the liver parenchyma, and the adjacent non-tumor tissues of ICC are normal liver tissues. Immunohistochemical staining of the ICC tissues showed KCa3.1 protein was mainly located in the cell membrane and cytoplasm (Fig. 1A). 
During the 81 ICC tissues, 52 (64.2\%) showed high KCa3.1 expression (KCa3.1+++ or $\mathrm{KCa} 3.1++$ ), while 29 (35.8\%) presented with low KCa3.1 expression (KCa3.1+ or KCa3.1-). Contrasting to ICC tissues, paracarcinoma tissues almost showed weak or negative KCa3.1 expression. We further analyzed the correlation between the KCa3.1 expression and some clinicopathological parameters of ICC patients. The KCa3.1 expression was significantly associated with age $(P=0.012)$, lymph node metastasis $(P=0.009)$, and TNM stage $(P=0.017)$. However, the KCa3.1 expression did not statistically differ by gender, serum AFP, serum CA19-9, tumor number, liver cirrhosis, macrovascular invasion, tumor size, Edmonson grade, and distant metastasis (Table 1).

Among the 81 ICC patients, the follow-up data of 14 ICC patients were incomplete and were therefore removed from the survival analysis (Fig. 1B). Kaplan-Meier analysis revealed ICC patients with high KCa3.1 expression had a worse overall survival than those with low KCa3.1 expression $(\mathrm{N}=67$,
$P=0.087$ ). The median survival time of ICC patients with low KCa3.1 expression was 23 months, whereas high KCa3.1 expression showed a shortened median survival time of 17 months.

\section{KCa3.1 channel inhibition blocks growth of ICC cell lines}

TRAM-34 is a specific inhibitor of KCa3.1 channel. Treatment of the ICC cells with TRAM-34 $(5-80 \mu \mathrm{M})$ for $24-72 \mathrm{~h}$ induced a reduced cellular proliferation in a concentration dependent manner. Two ICC cell lines showed different proliferative inhibition with TRAM-34 treatment. Both cell lines responded to $10 \mu \mathrm{M}$ TRAM-34 with a slight inhibition of their proliferation rate. The 50\% inhibition concentration (IC50) of TRAM-34 in Huh28 cells was about $36 \mu \mathrm{M}$ after 48 hours; while the IC50 value of TRAM-34 in HUCCT1 cells was about $52 \mu \mathrm{M}$ after 48 hours (Fig. 2A and 2B). Besides, the proliferation inhibiting effects of TRAM-34 in two ICC cell lines were not time dependent between $24 \mathrm{~h}, 48 \mathrm{~h}$ and $72 \mathrm{~h}$.
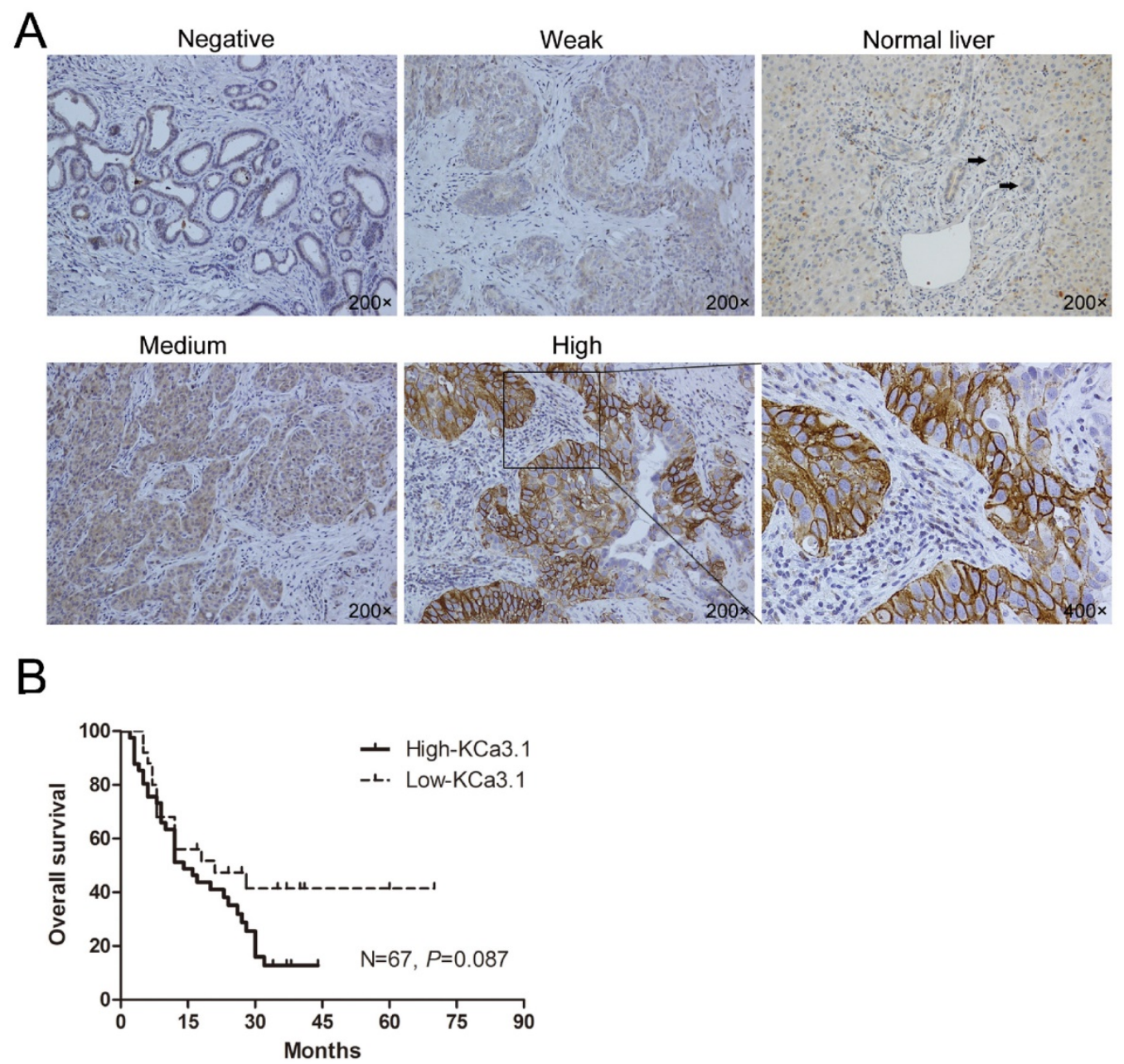

Figure 1. (A) Representative immunohistochemical pictures showing KCa3.1 channel protein in ICC tumors and normal intrahepatic bile duct (Negative, Weak, Normal liver, Medium, High: with 200× magnification; The black arrows indicate the normal intrahepatic bile duct in "Normal Liver" picture; The last graph is the partial enlargement of high KCa3.1 expression graph at $400 \times$ magnification). (B) Kaplan-Meier curve depicting overall survival according to the KCa3.1 channel protein expression pattern in ICC tissues $(n=67)$. 
Table 1. Relationship between $\mathrm{KCa} 3.1$ protein expression and clinicopathological features of 81 patients with ICC

\begin{tabular}{|c|c|c|c|}
\hline \multirow[t]{2}{*}{ Variables } & \multicolumn{2}{|c|}{ KCa3.1 density } & \multirow[t]{2}{*}{$\mathrm{p}$ Value } \\
\hline & Low-KCa3.1 & High-KCa3.1 & \\
\hline All patients & 29 & 52 & \\
\hline \multicolumn{4}{|l|}{ Age (years) } \\
\hline$<60$ & 19 & 19 & $0.012^{*}$ \\
\hline$\geq 60$ & 10 & 33 & \\
\hline \multicolumn{4}{|l|}{ Gender } \\
\hline Male & 16 & 32 & 0.576 \\
\hline Female & 13 & 20 & \\
\hline \multicolumn{4}{|c|}{ Serum AFP $(\mu \mathrm{g} / \mathrm{ml})$} \\
\hline$<20$ & 28 & 50 & 1.000 \\
\hline$\geq 20$ & 1 & 2 & \\
\hline \multicolumn{4}{|c|}{ Serum CA19-9 (37 U/ml) } \\
\hline$<37$ & 6 & 16 & 0.328 \\
\hline$\geq 37$ & 23 & 36 & \\
\hline \multicolumn{4}{|c|}{ Tumor number } \\
\hline Single & 23 & 36 & 0.328 \\
\hline Multiple & 6 & 16 & \\
\hline \multicolumn{4}{|c|}{ Liver cirrhosis } \\
\hline Absent & 26 & 45 & 0.955 \\
\hline Present & 3 & 7 & \\
\hline \multicolumn{4}{|c|}{ Macrovascular invasion } \\
\hline Absent & 24 & 39 & 0.421 \\
\hline Present & 5 & 13 & \\
\hline \multicolumn{4}{|c|}{ Tumor size $(\mathrm{cm})$} \\
\hline$<5$ & 10 & 9 & 0.080 \\
\hline$\geq 5$ & 19 & 43 & \\
\hline \multicolumn{4}{|c|}{ Edmonson grade } \\
\hline $\mathrm{I}+\mathrm{II}$ & 8 & 12 & 0.652 \\
\hline III+IV & 21 & 40 & \\
\hline \multicolumn{4}{|c|}{ Lymph node metastasis } \\
\hline Absent & 21 & 22 & $0.009^{* *}$ \\
\hline Present & 8 & 30 & \\
\hline \multicolumn{4}{|c|}{ Distant metastasis } \\
\hline Absent & 24 & 38 & 0.324 \\
\hline Present & 5 & 14 & \\
\hline \multicolumn{4}{|l|}{ TNM stage } \\
\hline I+II & 18 & 18 & $0.017^{*}$ \\
\hline III+IV & 11 & 34 & \\
\hline
\end{tabular}

${ }^{*}(p<0.05) ;{ }^{* *}(p<0.01)$

\section{KCa3.I channel blockade reduces ICC cell lines invasion}

The effects of TRAM-34 on migration and invasion of ICC cells were evaluated by transwell assay. The migratory potential of Huh2 28 cells and HUCCT1 cells were not affected under the treatment of TRAM-34 at a concentration of $20 \mu \mathrm{M}$ compared with DMSO treatment (Fig. 2C). However, TRAM-34 had a significant inhibitory effect on invasion in Huh28 and HUCCT1 cells (Fig. 3A). The numbers of invasive cells were decreased with TRAM-34 $20 \mu \mathrm{M}$ treatment in Huh28 and HUCCT1 cells, compared with DMSO treatment. To further evaluate these effects, we detected the protein expression of matrix metalloproteinases (MMPs) in these cells under the treatment of TRAM-34 or DMSO. We found expressions of MMP-2 and MMP-9 were markedly reduced under the treatment of 10,20 and $40 \mu \mathrm{M}$ TRAM-34 in Huh28 cells, compared with control group (Fig. 3B). And the expression of MMP-2 had no obvious change between the TRAM-34 treatment group and control group in HUCCT cells, while the expression of MMP-9 were decreased as the concentration of TRAM-34 elevated (Fig. 3C).

\section{Inhibition of $\mathrm{KCa} 3.1$ channel decreases the intracellular $\mathrm{Ca}^{2+}$ concentration in ICC cells}

The intracellular $\mathrm{Ca}^{2+}$ concentration was measured by Confocal Microscope. Before measurement, ICC cells (Huh28 cells and HUCCT1 cells) were treated with $20 \mu \mathrm{M}$ TRAM-34 or equivalent DMSO. Initially, the baseline values of $\left[\mathrm{Ca}^{+}\right]_{\mathrm{i}}$ were measured under $\mathrm{Ca}^{2+}$ free solution, then cells were treated with $5 \mathrm{mM} \mathrm{Ca}^{2+}$ solution. On adding $5 \mathrm{mM}$ $\mathrm{Ca}^{2+},\left[\mathrm{Ca}^{+}\right]_{\mathrm{i}}$ rose immediately in both two cell lines. Compared to control group, TRAM-34 could reduce the cellular $\mathrm{Ca}^{2+}$ uptake when giving $5 \mathrm{mM} \mathrm{Ca}^{2+}$ solution in Huh28 cells and HUCCT1 cells (Fig. 4).

\section{Knockdown of $\mathrm{KCa} 3.1$ reduces the proliferation, migration and invasion of ICC cell lines}

The expression of KCa3.1 was silenced in Huh28 and HUCCT1 cells by KCa3.1 siRNA. Knockdown of KCa3.1 in Huh28 and HUCCT1 cells were confirmed by Western blot (Fig. 5A). Cell proliferation assay showed that KCa3.1 silencing significantly decreased the viability of Huh28 and HUCCT1 cells compared to control group $(P<0.05$, Fig. 5B and 5C). The effect of KCa3.1 silencing on the migration and invasion of ICC cells was evaluated using the Matrigel model. We found that knockdown of KCa3.1 significantly decreased the migratory and invasive potential of Huh28 cells and HUCCT1 cells $(P<0.01$, Fig. 5D and $5 \mathrm{E})$. Further, we detected the expression of nuclear phospho-NF-kB p65 (active form of NF- $\mathrm{kB}$ ) in Huh28 cells and HUCCT1 cells when transfected with KCa3.1 siRNA. The results showed that the level of phospho-NF-kB p65 in nuclei was obviously decreased in HUCCT1 cells and reduced (not significantly) in Huh28 cells after KCa3.1 knockdown, compared to control group (Fig. 6).

\section{KCa3.1 channel blockade reduced tumor growth in nude mice}

Finally, we detected the effect of KCa3.1 channel blockade on tumor growth in vivo. Huh28 tumor-bearing mice were randomly administered with senicapoc or solvent (DMSO). Senicapoc is a KCa3.1 channel blocker which is similar to TRAM-34 in chemical structure, but with a better metabolic stability [15]. The tumor growth curves of senicapoc treatment group and control group in nude mice are shown in figure 7C. The tumor volume was 
significantly reduced after 6 days of senicapoc treatment compared to control group. At the end of the experiment, the mice were killed and the subcutaneous xenograft tumors were isolated and weighed. The average tumor weight of senicapoc treatment group was significantly lighter than control group ( $\mathrm{n}=10, P<0.05$, Fig. 7A and $7 \mathrm{~B}$ ). Further, we performed H\&E staining and Ki-67 immunohistochemistry of the respective tumors in two groups (Fig. 7D). Ki-67 protein was almost expressed in all tumor cells in two groups. But the staining intensity of Ki-67 was much lighter in senicapoc treatment group than control group, which indicated senicapoc decreased the proliferation of tumor cells in mice. These in vivo observations suggested the potential use of KCa3.1 inhibitors in the treatment of ICC.

\section{Discussion}

Intrahepatic cholangiocarcinoma (ICC) is a highly malignant cancer arising from the bile ducts in the liver with a poor prognosis [1]. In this study, we have demonstrated for the first time that potassium channel KCa3.1 was involved in the progression of intrahepatic cholangiocarcinoma. Pharmacological inhibition of KCa3.1 or knockdown of KCa3.1 could reduce the proliferation and invasion of ICC cells. Furthermore, in vivo experiments confirmed the anti-tumor efficacy of KCa3.1 inhibition.

Ion channels are transmembrane proteins facilitating the transport of ions across biological membranes. Recent studies found that ion channels were aberrantly expressed in many types of human cancers and played important roles in tumor progression [16-19]. Ion channels were involved in several cellular functions, such as proliferation, apoptosis, angiogenesis, migration, and invasion, mainly through regulating two important cellular parameters, the intracellular $\mathrm{Ca}^{2+}$ concentration and the cell volume $[20,21]$. $\mathrm{Ca}^{2+}$-activated potassium channels are voltage independent and are activated by changes in the intracellular calcium ion concentration. The KCa3.1 channel belongs to the $\mathrm{Ca}^{2+}$-activated potassium channel superfamily. Several studies have found in various human cancers including glioblastoma, breast adenocarcinoma, lung cancer, colorectal cancer, and renal carcinoma that KCa3.1 expression correlated with malignancy [5-9]. Besides, KCa3.1 has attracted attention as a prognostic biomarker in patients diagnosed with lung cancer or renal carcinoma $[5,13]$. However, so far, there is no information on the role of KCa3.1 in ICC progression.
A
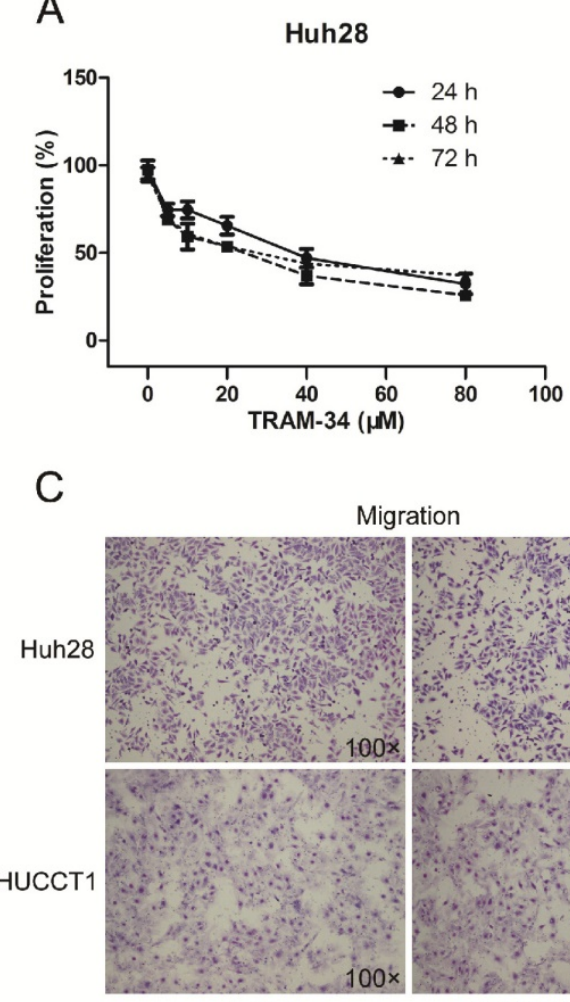

Control
Migration

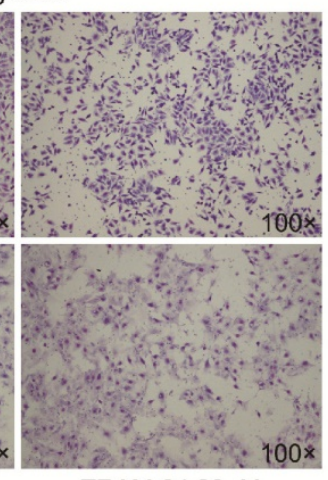

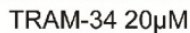

B
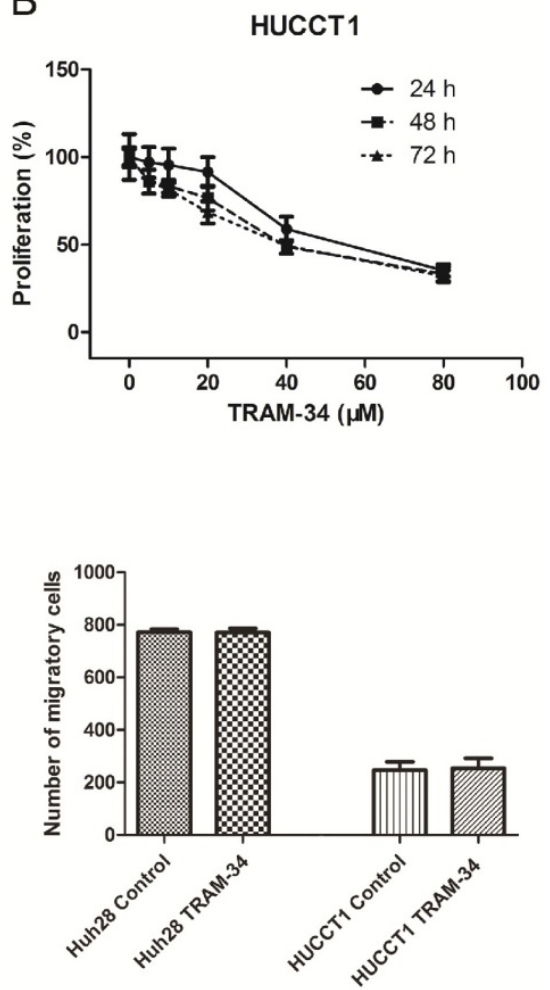

Figure 2. KCa3.1 channel inhibition blocks growth of ICC cell lines. (A) KCa3.1 channel inhibitor TRAM-34 blocks the growth of Huh28 cells with different concentrations $(0,5,10,20,40$, and $80 \mu \mathrm{M})$ at 24, 48, and 72 hour. (B) KCa3.1 channel inhibitor TRAM-34 blocks the growth of HUCCT1 cells with different concentrations $(0,5,10,20,40$, and $80 \mu \mathrm{M})$ at 24,48 , and 72 hour. (C) Effects of KCa3.1 channel inhibitor on migration in ICC cell lines. Left panels are representative images of migration of Huh28 and HUCCT1 cells using the transwell chamber (100x magnification). Right panels are the comparison of migratory cell numbers. Data are mean \pm SD . 
A
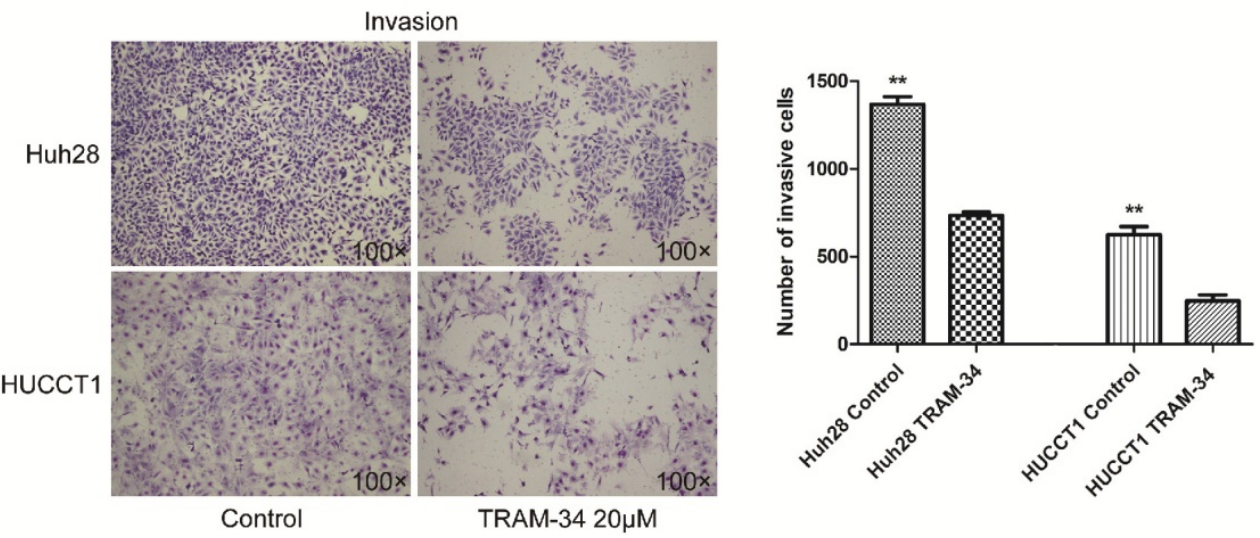

B

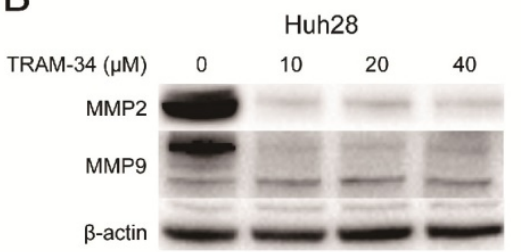

C

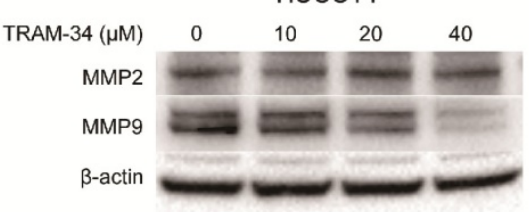

Figure 3. Effects of $\mathrm{KCa} 3.1$ channel inhibitor on invasion in ICC cell lines. (A) Left panels are representative images of invasion of Huh28 and HUCCTl cells using the Matrigel model (100× magnification). Right panels are the comparison of invasive cell numbers. Data are mean \pm SD, $* * P<0.01$. (B) Effects of TRAM-34 on protein expressions of MMP-2 and MMP-9 in Huh28 cells when treated with increasing doses of TRAM-34. (C) Effects of TRAM-34 on protein expressions of MMP-2 and MMP-9 in HUCCT1 cells when treated with increasing doses of TRAM-34, beta-actin serves as an internal reference.
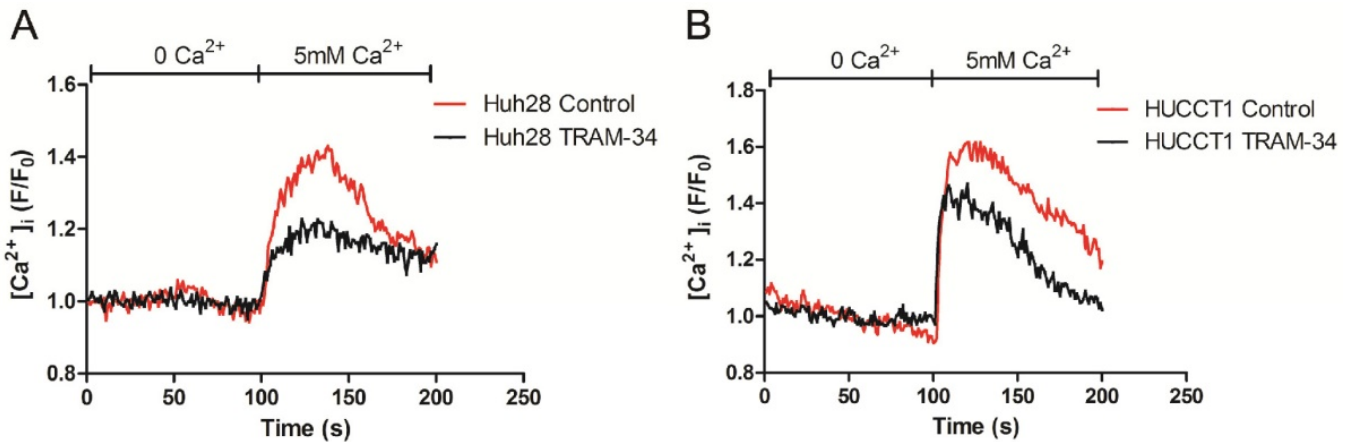

Figure 4. $\mathrm{KCa} 3.1$ channel inhibitor reduces the intracellular $\mathrm{Ca}^{2+}$ concentration in Huh28 cells (A) and HUCCTI cells (B).

Our study showed that KCa3.1 channels were highly expressed in $64.2 \%$ primary intrahepatic cholangiocarcinoma tissues compared to normal paracarcinoma tissues ( $\mathrm{N}=81)$. And high KCa3.1 expression not only significantly associated with age, lymph node metastasis and TNM stage, but also indicated a poor overall survival in patients. Thus, KCa3.1 channel may be a potential predictor of poor outcome in ICC patients.

TRAM-34, derivative of clotrimazole, is a well-known blocker to study the roles of KCa3.1 channels. It has reported that TRAM-34 could effectively inhibit proliferation and motility in several types of human cancers including hepatocellular carcinoma, glioblastoma and lung cancer $[7,9,22]$. Consistent with these findings, our study showed that TRAM-34 could block the proliferation of two ICC cells. The anti-proliferation efficacy was dose-dependent rather than time-dependent. To explain the proliferation inhibitory effects of TRAM-34, we first examined the apoptotic levels in TRAM-34 treated cells by flow cytometry and Western blot (data not shown). But there was no obvious induction of apoptosis upon treatment of Huh28 cells and HUCCT1 cells with TRAM-34. Then we measured the intracellular calcium concentration in ICC cell lines with and without inhibition. The data showed TRAM-34 could reduce the cellular $\mathrm{Ca}^{2+}$ uptake after $5 \mathrm{mM} \mathrm{Ca}^{2+}$ stimulus. Several studies have reported that the activity of KCa3.1 channels facilitates the $\mathrm{Ca}^{2+}$ influx through setting the cell membrane potential and the driving force for $\mathrm{Ca}^{2+}$ influx in different cell types [23-25]. A rise in cytosolic $\mathrm{Ca}^{2+}$ concentration plays a critical role in mitogen-mediated cell growth $[25,26]$. Thus, the anti-proliferation effects of TRAM-34 in our study are likely to be due to the reduction of intracellular calcium ion in ICC cell lines. 
A

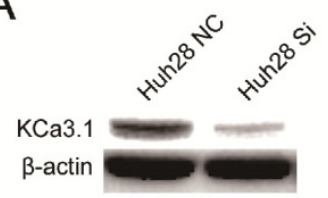

B
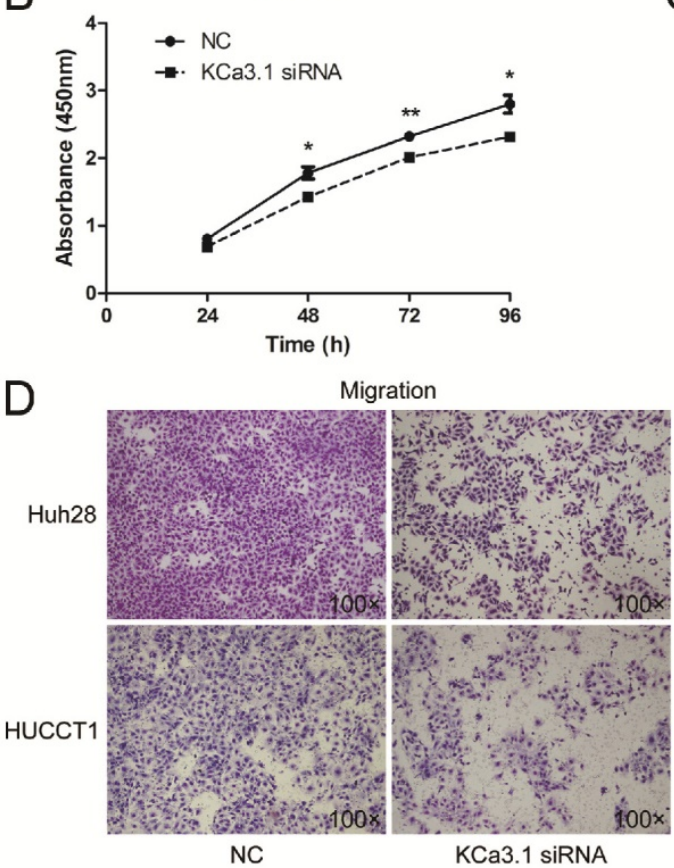

E

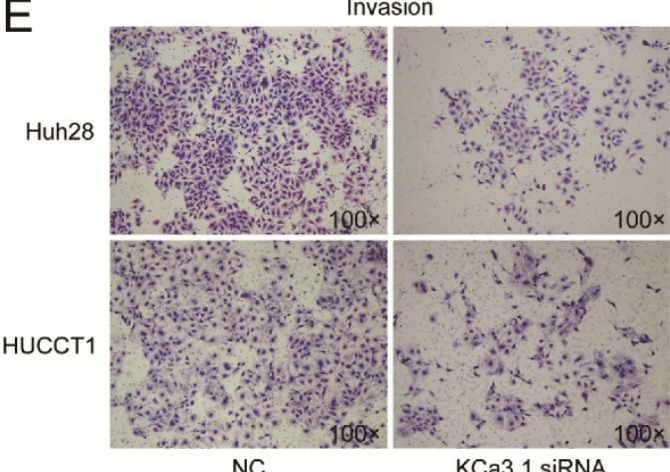

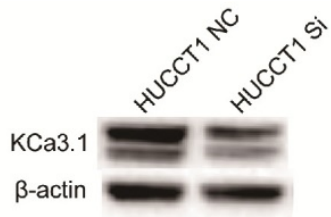

C

HUCCT1
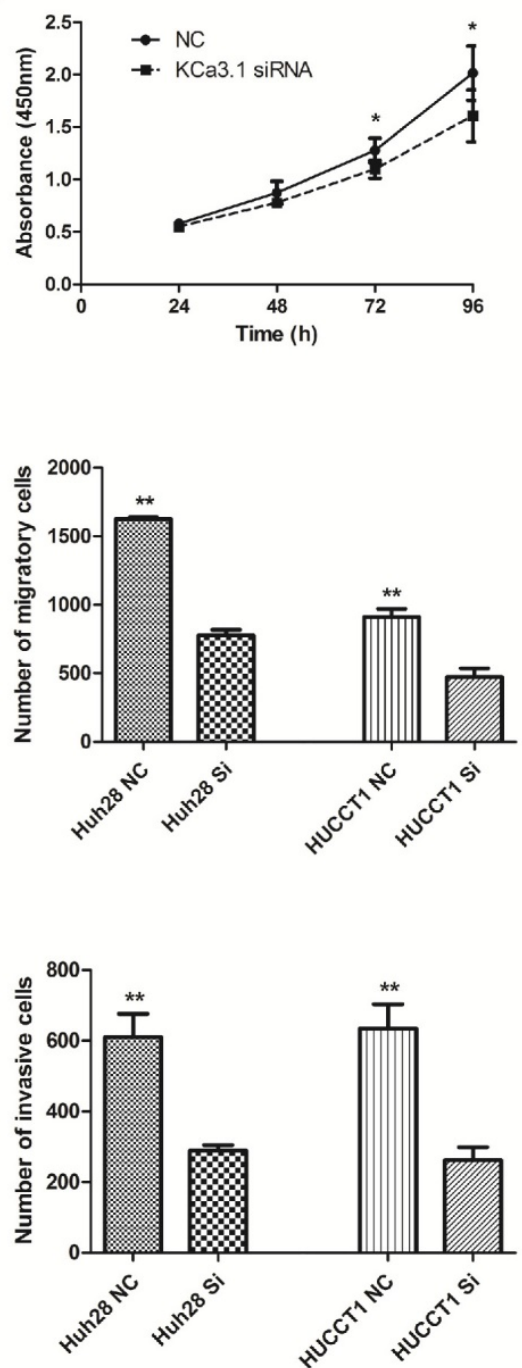

Figure 5. Effects of KCa3.1 siRNA on the proliferation, migration and invasion of ICC cell lines. (A) Protein levels of KCa3.1 channel in Huh28 and HUCCT1 cells transfected with $\mathrm{KCa} 3.1$ siRNA were accessed by Western blot and normalized to beta-actin. (B) Knockdown of $\mathrm{KCa} 3.1$ decreases the viability of Huh28 cells ( ${ }^{* P}<$ 0.05). (C) Knockdown of KCa3.1 decreases the viability of HUCCTI cells $(* P<0.05)$. (D) Knockdown of KCa3.1 significantly decreased the migratory potential of Huh28 and HUCCT1 cells (**P $<0.01,100 \times$ magnification). (E) Knockdown of KCa3.1 significantly decreased the invasive potential of Huh28 and HUCCTI cells $(* * P$ $<0.01,100 \times$ magnification).

We further studied whether blockade of KCa3.1 channel may cause the inhibition of migration and invasion of ICC cells. To reduce the anti-proliferative effect of TRAM-34 on ICC cell lines as possible, we chose the TRAM-34 at $20 \mu \mathrm{M}$ which was near to the IC20 value of TRAM-34 to perform migration and invasion assays in two ICC cell lines. The results indicated that TRAM-34 had no impact on migratory potential but had an inhibitory effect on invasive potential of ICC cell lines.

Previous studies have reported KCa3.1 channel inhibitor TRAM-34 weakened the migration of endometrial carcinoma cells, hepatocellular carcinoma cells and pancreatic stellate cells [22, 27, 28]. Conversely, a recent study found TRAM-34 could increase intracellular calcium concentration and stimulate cell migration in pancreatic cancer [29]. This might be due to different experimental conditions, such as cell line, drug concentration and duration etc. In our study, TRAM-34 significantly decreased the protein expressions of MMP-2 and MMP-9 in Huh28 cells, and reduced the protein expressions of MMP-9 in HUCCT1 cells as the drug concentration elevated. 


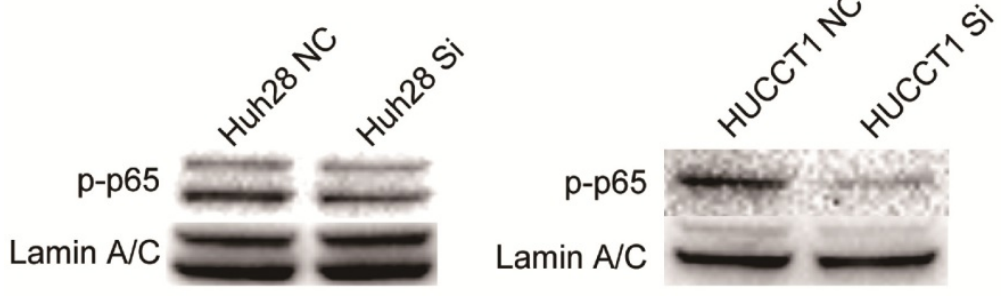

Figure 6. Knockdown of KCa3.1 reduces the activation of NF-KB in Huh28 cells and HUCCT1 cells. Western blot detected the expression of phospho-NF-KB p65 in the nuclear fractions of Huh28 and HUCCT1 cells transfected with KCa3.1 siRNA, Lamin A/C was chosen for the internal reference of nuclear protein.
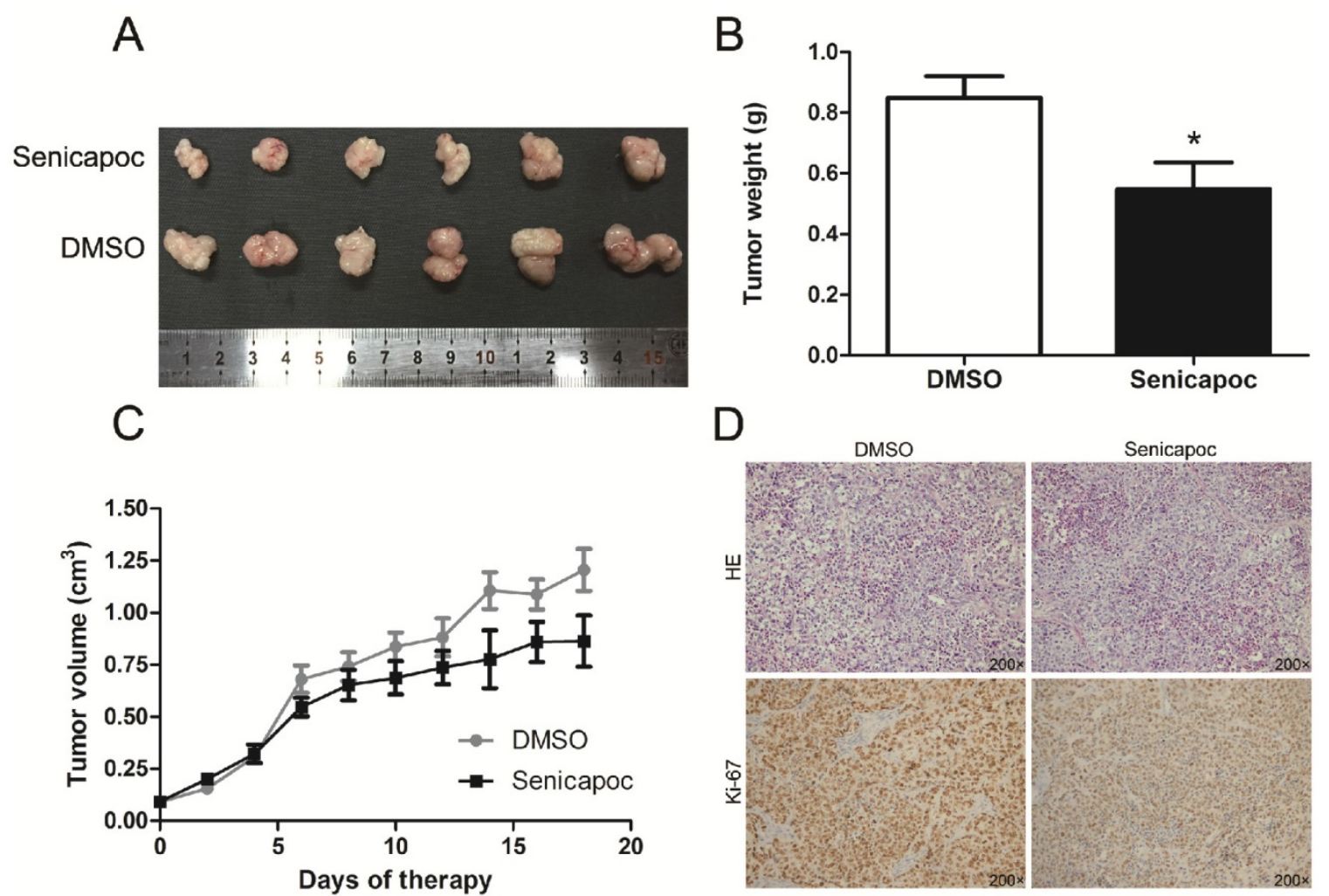

Figure 7. $\mathrm{KCa} 3.1$ blocker senicapoc impairs growth of tumors derived from Huh28 cells in vivo. (A) Images show the features of tumor growth in senicapoc group and DMSO group at necropsy. (B) Tumor weight when the tumors were harvested $(* P<0.05)$. (C) The tumor volume of senicapoc-treated $(n=10)$ and DMSO-treated $(n=10)$ tumors was calculated by caliper measurements. (D) Representative images of H\&E staining and Ki-67 immunohistochemistry of the tumor (200× magnification). Upper panels, H\&E staining; lower panels, immunostaining.

It's well-known that MMP-2 and MMP-9 play pivotal roles in the degradation of the extracellular matrix. Our data imply that KCa3.1 might enhance the invasion of ICC cells through pathways involving MMP-2 and MMP-9, but the exact molecular mechanism involved needs further investigations.

In addition, KCa3.1 siRNA was used to study the roles of KCa3.1 in ICC. Our results demonstrated that knockdown of KCa3.1 reduced the proliferation, migration and invasion of Huh28 cells and HUCCT1 cells. As for underlying relevant mechanisms, here we examined effect of KCa3.1 knockdown on the activation of the nuclear transcription factor NF-kB. NF- $\mathrm{kB}$ consists of a family of transcription factors which are involved in the regulation of various biological responses [30]. NF- $\mathrm{kB}$ regulates the expression of genes related to many processes that play an important role in cancer development and progression, such as apoptosis, proliferation and migration. Previous studies have reported that constitutive or aberrant NF-KB activation has been identified in some human malignancies, including hepatocellular carcinoma, colorectal carcinoma and pancreatic adenocarcinoma [31-33]. Our data showed that knockdown of $\mathrm{KCa} 3.1$ obviously reduced the activation of NF-KB in HUCCT1 cells, while the reduction of activation of NF-kB in Huh28 cells was not significant as HUCCT1 cells. And the decreased NF-kB activation may have contributed to the anti-tumor effects of KCa3.1 knockdown.

Senicapoc was a KCa3.1 channel blocker which was similar to TRAM-34 in chemical structure, but with a better metabolic stability [15]. Thus, senicapoc was used to explore the anti-tumor effects of KCa3.1 
channel blockade in vivo in our experiments. It has been reported that the tumor growth of NSCLC cells in mice could be significantly inhibited with the treatment of KCa3.1 blocker senicapoc [9]. Consistently, our data showed KCa3.1 blockade with senicapoc reduced the ICC tumor growth in nude mice.

Taken together, our experiments showed that the protein expression of $\mathrm{KCa} 3.1$ channel was upregulated in ICC tumors and was correlated with age, lymph node metastasis, and TNM stage. A high KCa3.1 expression indicated a worse prognosis in ICC patients. Blocking KCa3.1 channel with the specific inhibitor TRAM-34 reduced the proliferation and invasion of ICC cells. Knockdown of KCa3.1 could achieve the same effects through decreasing NF-KB activation. Additionally, in vivo studies demonstrated that KCa3.1 blockade reduced ICC tumor growth. These observations suggested KCa3.1 might be a promising therapeutic target in intrahepatic cholangiocarcinoma.

\section{Abbreviations}

KCa3.1: The intermediate conductance calcium-activated potassium channel; ICC: intrahepatic cholangiocarcinoma; $\left[\mathrm{Ca}^{2+}\right]_{\mathrm{i}}$ : intracellular $\mathrm{Ca}^{2+}$ concentration; MMPs: matrix metalloproteinases.

\section{Acknowledgements}

We thank the excellent technical assistant of Lin Zhao Xiaonan from Imaging Facility, Core Facilities, Zhejiang University School of Medicine with the confocal laser microscopy.

\section{Funding}

This work was supported by the Major Research Plan of National Natural Science Foundation of China (91542205) to SZ, the National Natural Science Foundation of China (81272675 and 81570575) to PS, the National Natural Science Foundation of China (31440053) to BT, the Natural Science Foundation of Zhejiang Province (LY15H030005) to PS, and Innovative Research Groups of the National Natural Science Foundation of China (81421062). The funders had no role in study design, data collection and analysis, decision to publish or preparation of the manuscript.

\section{Competing Interests}

The authors have declared that no competing interest exists.

\section{References}

1. Sia D, Tovar V, Moeini A, Llovet JM. Intrahepatic cholangiocarcinoma: pathogenesis and rationale for molecular therapies. Oncogene. 2013; 32: 4861-70.
2. Bergquist A, von Seth E. Epidemiology of cholangiocarcinoma. Best practice \& research Clinical gastroenterology. 2015; 29: 221-32

3. Rizvi S, Gores GJ. Pathogenesis, diagnosis, and management of cholangiocarcinoma. Gastroenterology. 2013; 145: 1215-29.

4. Catacuzzeno L, Fioretti B, Franciolini F. Expression and Role of the Intermediate-Conductance Calcium-Activated Potassium Channel KCa3.1 in Glioblastoma. Journal of signal transduction. 2012; 2012: 421564.

5. Rabjerg M, Olivan-Viguera A, Hansen LK, Jensen L, Sevelsted-Moller L, Walter S, et al. High Expression of KCa3.1 in Patients with Clear Cell Renal Carcinoma Predicts High Metastatic Risk and Poor Survival. Plos One. 2015; 10: e0122992.

6. Lai W, Liu L, Zeng Y, Wu H, Xu H, Chen S, et al. KCNN4 Channels participate in the EMT induced by PRL-3 in colorectal cancer. Medical Oncology. 2013; 30: 566.

7. D'Alessandro G, Catalano M, Sciaccaluga M, Chece G, Cipriani R, Rosito M, et al. KCa3.1 channels are involved in the infiltrative behavior of glioblastoma in vivo. Cell Death \& Disease. 2013; 4: e773.

8. Haren N, Khorsi H, Faouzi M, Ahidouch A, Sevestre H, Ouadid-Ahidouch H. Intermediate conductance $\mathrm{Ca} 2+$ activated $\mathrm{K}+$ channels are expressed and functional in breast adenocarcinomas: correlation with tumour grade and metastasis status. Histology and Histopathology. 2010; 25: 1247-55.

9. Bulk E, Ay AS, Hammadi M, Ouadid-Ahidouch H, Schelhaas S, Hascher A, et al. Epigenetic dysregulation of KCa 3.1 channels induces poor prognosis in lung cancer. Int J Cancer. 2015; 137: 1306-17.

10. Di L, Srivastava S, Zhdanova O, Ding Y, Li Z, Wulff H, et al. Inhibition of the $\mathrm{K}+$ channel KCa3.1 ameliorates T cell-mediated colitis. Proceedings of the National Academy of Sciences of the United States of America. 2010; 107: 1541-6.

11. Toyama K, Wulff H, Chandy KG, Azam P, Raman G, Saito T, et al. The intermediate-conductance calcium-activated potassium channel KCa3.1 contributes to atherogenesis in mice and humans. The Journal of clinical investigation. 2008; 118: 3025-37.

12. Freise C, Ruehl M, Seehofer D, Hoyer J, Somasundaram R. The inhibitor of Ca2+-dependent $\mathrm{K}+$ channels TRAM-34 blocks growth of hepatocellular carcinoma cells via downregulation of estrogen receptor alpha mRNA and nuclear factor-kappaB. Investigational New Drugs. 2013; 31: 452-7.

13. Bulk E, Ay A-S, Hammadi M, Ouadid-Ahidouch H, Schelhaas S, Hascher A, et al. Epigenetic dysregulation of $\mathrm{K}(\mathrm{Ca}) 3.1$ channels induces poor prognosis in lung cancer. International Journal of Cancer. 2015; 137: 1306-17.

14. Wulff H, Miller MJ, Hansel W, Grissmer S, Cahalan MD, Chandy KG. Design of a potent and selective inhibitor of the intermediate-conductance Ca2+-activated $\mathrm{K}+$ channel, IKCa1: a potential immunosuppressant. Proceedings of the National Academy of Sciences of the United States of America. 2000; 97: 8151-6.

15. Maezawa I, Jenkins DP, Jin BE, Wulff H. Microglial KCa3.1 Channels as a Potential Therapeutic Target for Alzheimer's Disease. International journal of Alzheimer's disease. 2012; 2012: 868972.

16. Fraser SP, Diss JK, Chioni AM, Mycielska ME, Pan $\mathrm{H}$, Yamaci RF, et al. Voltage-gated sodium channel expression and potentiation of human breast cancer metastasis. Clinical cancer research : an official journal of the American Association for Cancer Research. 2005; 11: 5381-9.

17. Wadhwa S, Wadhwa P, Dinda AK, Gupta NP. Differential expression of potassium ion channels in human renal cell carcinoma. International urology and nephrology. 2009; 41: 251-7.

18. Britschoi A Bill A, Brinkhaus $\mathrm{H}$, Rothwell $\mathrm{C}$, Clay $\mathrm{I}$ Duss $\mathrm{S}$, et al. Calcium-activated chloride channel ANO1 promotes breast cancer progression by activating EGFR and CAMK signaling. Proceedings of the National Academy of Sciences of the United States of America. 2013; 110: E1026-34.

19. Rybarczyk P, Gautier M, Hague F, Dhennin-Duthille I, Chatelain D, Kerr-Conte J, et al. Transient receptor potential melastatin-related 7 channel is overexpressed in human pancreatic ductal adenocarcinomas and regulates human pancreatic cancer cell migration. Int J Cancer. 2012; 131: E851-61.

20. Arcangeli A, Crociani O, Lastraioli E, Masi A, Pillozzi S, Becchetti A. Targeting ion channels in cancer: a novel frontier in antineoplastic therapy. Current medicinal chemistry. 2009; 16: 66-93.

21. Kunzelmann K. Ion channels and cancer. The Journal of membrane biology. 2005; 205: 159-73.

22. Liu Y, Zhao L, Ma W, Cao X, Chen H, Feng D, et al. The Blockage of KCa3.1 Channel Inhibited Proliferation, Migration and Promoted Apoptosis of Human Hepatocellular Carcinoma Cells. Journal of Cancer. 2015; 6: 643-51.

23. Bi D, Toyama K, Lemaitre V, Takai J, Fan F, Jenkins DP, et al. The intermediate conductance calcium-activated potassium channel $\mathrm{KCa} 3.1$ regulates vascular smooth muscle cell proliferation via controlling calcium-dependent signaling. The Journal of biological chemistry. 2013; 288: 15843-53.

24. Wang $\mathrm{J}, \mathrm{Xu}$ YQ, Liang $\mathrm{YY}$, Gongora $\mathrm{R}$, Warnock DG, Ma HP. An intermediate-conductance $\mathrm{Ca}(2+)$-activated $\mathrm{K} \quad\left(^{+}\right)$channel mediates $\mathrm{B}$ lymphoma cell cycle progression induced by serum. Pflugers Archiv European journal of physiology. 2007; 454: 945-56.

25. Faouzi M, Hague F, Geerts D, Ay AS, Potier-Cartereau M, Ahidouch A, et al. Functional cooperation between KCa3.1 and TRPC1 channels in human breast cancer: Role in cell proliferation and patient prognosis. Oncotarget. 2016; 7: 36419-35.

26. Golovina VA, Platoshyn O, Bailey CL, Wang J, Limsuwan A, Sweeney M, et al. Upregulated TRP and enhanced capacitative $\mathrm{Ca}(2+)$ entry in human 
pulmonary artery myocytes during proliferation. American journal of physiology Heart and circulatory physiology. 2001; 280: H746-55.

27. Zhang Y, Feng Y, Chen L, Zhu J. Effects of Intermediate-Conductance $\mathrm{Ca} 2+$-Activated $\mathrm{K}+$ Channels on Human Endometrial Carcinoma Cells. Cell Biochemistry and Biophysics. 2015; 72: 515-25.

28. Storck H, Hild B, Schimmelpfennig S, Sargin S, Nielsen N, Zaccagnino A, et al. Ion channels in control of pancreatic stellate cell migration. Oncotarget. 2016; 8: 769-84.

29. Bonito B, Sauter DRP, Schwab A, Djamgoz MBA, Novak I. KCa3.1 (IK) modulates pancreatic cancer cell migration, invasion and proliferation: anomalous effects on TRAM-34. Pflügers Archiv - European Journal of Physiology. 2016; 468: 1865-75.

30. Dolcet X, Llobet D, Pallares J, Matias-Guiu X. NF-kB in development and progression of human cancer. Virchows Archiv. 2005; 446: 475-82.

31. Wu JM, Sheng H, Saxena R, Skill NJ, Bhat-Nakshatri P, Yu M, et al. NF-kappaB inhibition in human hepatocellular carcinoma and its potential as adjunct to sorafenib based therapy. Cancer letters. 2009; 278: 145-55.

32. Kojima M, Morisaki T, Sasaki N, Nakano K, Mibu R, Tanaka M, et al. Increased nuclear factor-kB activation in human colorectal carcinoma and its correlation with tumor progression. Anticancer research. 2004; 24: 675-81.

33. Wang W, Abbruzzese JL, Evans DB, Larry L, Cleary KR, Chiao PJ. The nuclear factor-kappa B RelA transcription factor is constitutively activated in human pancreatic adenocarcinoma cells. Clinical cancer research : an official journal of the American Association for Cancer Research. 1999; 5: 119-27. 